\title{
SEARCH FOR DIRECT CP VIOLATION IN CHARGED KAON DECAYS FROM NA48/2 EXPERIMENT
}

\author{
S.Balev ${ }^{a}$ \\ Joint Institute for Nuclear Research, 141980 Dubna, Russia
}

\begin{abstract}
A high precision measurement of the asymmetry in Dalitz plot slopes $A_{g}=\left(g^{+}-g^{-}\right) /\left(g^{+}-g^{-}\right)$was performed by the experiment NA48/2. The obtained results, $A_{g}^{c}=(-1.5 \pm 2.1) \cdot 10^{-4}$ and $A_{g}^{n}=(1.8 \pm 1.8) \cdot 10^{-4}$ are based on record statistics $-\sim 3.1 \cdot 10^{9} K^{ \pm} \rightarrow \pi^{ \pm} \pi^{+} \pi^{-}$decays and $\sim 9.1 \cdot 10^{7} K^{ \pm} \rightarrow$ $\pi^{ \pm} \pi^{0} \pi^{0}$ decays, correspondingly. The precision of the measurement is one order of magnitude better than the previous experiments and is limited by the statistical error.
\end{abstract}

\section{Itroduction}

$C P$ violation plays an important role in elementary particles physics: on one hand, its studies allow to make precise tests to the Standard Model (SM) and to search for new physics; on the other hand, this phenomenon is in the core of the baryogenesis according to modern cosmological models. Therefore, studies of each possible manifestation of $C P$-violation are tasks of fundamental importance. In kaons, besides the $\epsilon^{\prime} / \epsilon$ parameter in $K^{0} \rightarrow \pi \pi$ decays, promising complementary observables are the rates of GIM-suppressed rare kaon decays proceeding through neutral currents, and the asymmetry between $K^{+}$and $K^{-}$ decays to three pions.

The $K^{ \pm} \rightarrow 3 \pi$ matrix element can be parameterized by a polynomial expansion in two Lorentz-invariant variables $u$ and $v$ :

$$
|M(u, v)|^{2} \propto 1+g u+h u^{2}+k v^{2}+\ldots
$$

where $|h|,|k| \ll|g|$ are the slope parameters, $u=\left(s_{3}-s_{0}\right) / m_{\pi}^{2}, v=\left(s_{1}-\right.$ $\left.s_{2}\right) / m_{\pi}^{2}$, where $m_{\pi}$ is the charged pion mass, $s_{i}=\left(p_{K}-p_{i}\right)^{2}, s_{0}=\sum s_{i} / 3$ $(i=1,2,3), p_{K}$ and $p_{i}$ are kaon and $i$-th pion 4-momenta, respectively. The index $i=3$ corresponds to the odd pion. The parameter of direct $C P$ violation is usually defined as: $A_{g}=\left(g^{+}-g^{-}\right) /\left(g^{+}+g^{-}\right)$, where $g^{+}$is the linear coefficient in (1) for $K^{+}$and $g^{-}-$for $K^{-}$. A deviation of $A_{g}$ from zero is a clear indication for direct $C P$ violation.

The experimental precision before NA48/2 for both decay modes, $K^{ \pm} \rightarrow$ $\pi^{ \pm} \pi^{+} \pi^{-}$and $K^{ \pm} \rightarrow \pi^{ \pm} \pi^{0} \pi^{0}$, is at the level of $10^{-3}$ [1], while SM predictions for $A_{g}$ are below $10^{-4}$ [2]. However, some theoretical calculations involving processes beyond the SM [3] predict substantial enhancements of the asymmetry, which could be observed in the present experiment. 


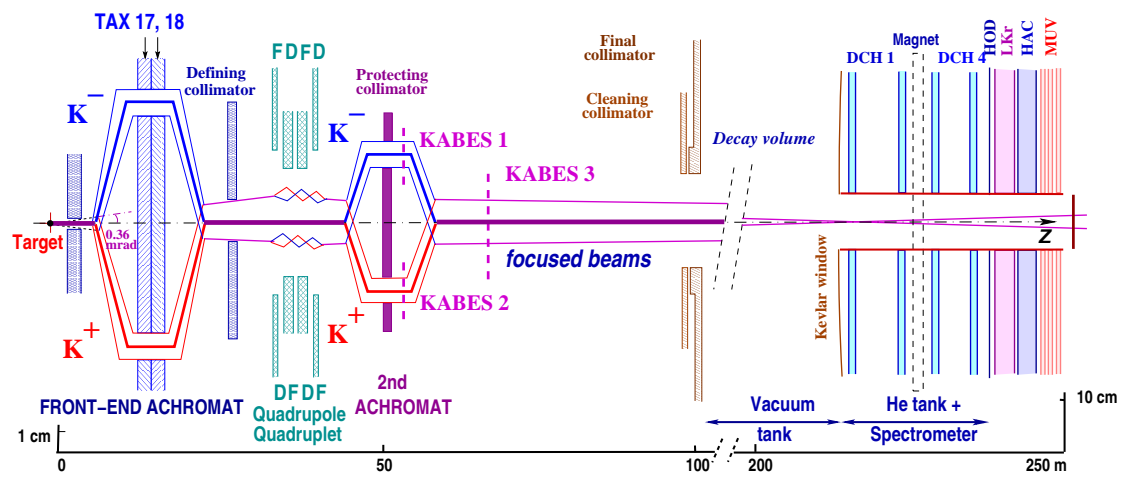

Figure 1: Schematic view of the NA48/2 experiment: beam line (TAX17,18: motorized beam dump/collimators used to select the momentum of the $K^{+}$and $K^{-}$beams; DFDF: focusing quadrupoles; KABES1-3: beam spectrometer stations), decay volume and detector (DCH1-4: drift chambers; Hodo: hodoscope; LKr: electromagnetic calorimeter; HAC: hadron calorimeter; MUV: muon veto).

\section{Experimental Setup}

The NA48/2 experiment at the CERN SPS was designed especially to search for direct $C P$ violation in the decays of charged kaons, and collected data in 2003 and 2004. The highest possible level of charge symmetry between $K^{+}$and $K^{-}$was a crucial requirement in the choice of beam, experimental apparatus, strategy of data taking and analysis in order to reach a high accuracy in the measurement of the charge asymmetry parameter $A_{g}$.

A novel beam line (Fig. 1) with two simultaneous charged beams of opposite charges was designed and built in the high intensity hall (ECN3) at the CERN SPS. The charged particle beams were produced by $400 \mathrm{GeV}$ SPS protons on a beryllium target. $K^{+}$and $K^{-}$with momentum $(60 \pm 3) \mathrm{GeV} / c$ were selected symmetrically by an achromatic magnet system ('achromat') which separates vertically the two beams and recombines them again on the same axis. Frequent inversion of the magnetic field polarities in all the beamline elements provides a high level of intrinsic cancelation of the possible systematic effects in the beam line.

The entire reconstruction of $K^{ \pm} \rightarrow \pi^{ \pm} \pi^{+} \pi^{-}$decays and the determination of the kaon charge in $K^{ \pm} \rightarrow \pi^{ \pm} \pi^{0} \pi^{0}$ decays rely on a magnetic spectrometer. Two drift chambers are located upstream and two downstream of a dipole magnet which deflects charged particles horizontally with a transverse momentum kick of $120 \mathrm{MeV} / c$. The magnetic field was reversed frequently in order to cancel possible left-right asymmetries in the detector system. The momen-

\footnotetext{
${ }^{a}$ e-mail: balev@sunse.jinr.ru
} 
tum resolution of the magnetic spectrometer is $\sigma(p) / p=1.0 \% \oplus 0.044 \% p(p$ in $\mathrm{GeV} / c)$. The acceptance of the spectrometer is defined mainly by an evacuated beam tube passing through its centre, with a diameter of $\sim 16 \mathrm{~cm}$, in which travel the surviving beam particles as well as the muons from $\pi \rightarrow \mu \nu$ decays.

The reconstruction of $K^{ \pm} \rightarrow \pi^{ \pm} \pi^{0} \pi^{0}$ decays is based mainly on the use of liquid krypton calorimeter ( $\mathrm{LKr}$ ), which measures the energies of the four photons from $\pi^{0}$ decays. The LKr has an energy resolution $\sigma(E) / E=0.032 / \sqrt{E} \oplus$ $0.09 / E \oplus 0.0042(E$ in $\mathrm{GeV})$ and spatial resolution for a single electromagnetic shower $\sigma_{x}=\sigma_{y}=0.42 / \sqrt{E} \oplus 0.06 \mathrm{~cm}$ for the transverse coordinates $x$ and $y$. The use of à priori charge symmetric detector helps to keep the result in $K^{ \pm} \rightarrow \pi^{ \pm} \pi^{0} \pi^{0}$ mode practically unbiased.

A hodoscope is used for precise time measurement of the charged particles and as a component in the trigger system for both decay modes. Detailed description of the detector components can be found elsewhere [4].

\section{Asymmetry measurement method}

The asymmetry measurement is based on the comparison of the $u$ spectra for $K^{+}$and $K^{-}$decays, $N^{+}(u)$ and $N^{-}(u)$, respectively. The ratio of the $u$ spectra $N^{+}(u) / N^{-}(u)$ is proportional to $\left[1+\Delta g \cdot u /\left(1+g u+h u^{2}\right)\right]$, where $g$ and $h$ are the actual of the Dalitz-slope parameters [5]. The possible presence of a direct $\mathrm{CP}$ violating difference between the linear slopes of $K^{+}$and $K^{-}, \Delta g=g^{+}-g^{-}$, can be extracted from a fit to this ratio. The measured asymmetry is then given by $A_{g}=\Delta g / 2 g$.

In order to minimize the effect of beam and detector asymmetries, we use the ratio $R_{4}(u)$, defined as a product of four $N^{+}(u) / N^{-}(u)$ ratios:

$$
R_{4}(u)=\frac{N_{A^{+} B^{+}}^{+}(u)}{N_{A^{+} B^{+}}^{-}(u)} \cdot \frac{N_{A^{+} B^{-}}^{+}(u)}{N_{A^{+} B^{-}}^{-}(u)} \cdot \frac{N_{A^{-} B^{+}}^{+}(u)}{N_{A^{-} B^{+}}^{-}(u)} \cdot \frac{N_{A^{-} B^{-}}^{+}(u)}{N_{A^{-} B^{-}}^{-}(u)},
$$

where the upper index corresponds to the kaon charge and lower index denotes the polarity in the beam-line $(A)$ and spectrometer magnet $(B)$. The parameter $\Delta g$ is extracted from a fit to the measured quadruple ratio $R_{4}(u)$ using the function $f(u)=R \cdot\left[1+\Delta g \cdot u /\left(1+g u+h u^{2}\right)\right]^{4}$. The measured slope difference is insensitive to the normalization parameter $R$, which reflects the ratio of $K^{+}$ and $K^{-}$fluxes $(\sim 1.8)$.

The quadruple ratio method complements the procedure of magnet polarity reversal. It allows cancelation at first order of systematic biases in the beam line and in the detector system. A reduction of possible systematic biases due to the presence of stray permanent magnetic fields is achieved by the radial cuts around the average beam position, which make the geometrical acceptance to particles azimuthally symmetric. The only residual sensitivity to instrumental charge asymmetries is associated with time variations of any 
acceptance asymmetries occurring on a time scale shorter than the magnetic field alternation period, which are studied carefully by a number of monitors recorded throughout data taking.

\section{Result and conclusions}

In total, $3.1 \cdot 10^{9} K^{ \pm} \rightarrow \pi^{ \pm} \pi^{+} \pi^{-}$and $91 \cdot 10^{6} K^{ \pm} \rightarrow \pi^{ \pm} \pi^{0} \pi^{0}$ decays were selected for the analysis. The result in terms of linear slope difference $\Delta g$ with only the statistical error quoted is $\Delta g^{c}=(0.7 \pm 0.7) \cdot 10^{-4}$ for $K^{ \pm} \rightarrow \pi^{ \pm} \pi^{+} \pi^{-}$ and $\Delta g^{n}=(2.2 \pm 2.1) \cdot 10^{-4}$ for $K^{ \pm} \rightarrow \pi^{ \pm} \pi^{0} \pi^{0}$ decay mode. These results are free of systematic biases in the first approximation due to the implemented method of cancellation of various apparatus imperfections. However, the checks of possible systematic contributions have been done, and corresponding uncertainties were obtained [6]. The obtained parameters $A_{g}$ are:

$$
\begin{gathered}
A_{g}^{c}=\left(-1.5 \pm 1.5_{\text {stat. }} \pm 1.4_{\text {syst. }}\right) \cdot 10^{-4}=(-1.5 \pm 2.1) \cdot 10^{-4}, \\
A_{g}^{n}=\left(1.8 \pm 1.7_{\text {stat. }} \pm 0.5_{\text {syst. }}\right) \cdot 10^{-4}=(1.8 \pm 1.8) \cdot 10^{-4}
\end{gathered}
$$

correspondingly for $K^{ \pm} \rightarrow \pi^{ \pm} \pi^{+} \pi^{-}$and $K^{ \pm} \rightarrow \pi^{ \pm} \pi^{0} \pi^{0}$ decay modes. The results are one order of magnitude more precise than previous measurements and are consistent with the predictions of the SM.

\section{References}

[1] W.T. Ford et al., Phys. Rev. Lett. 25, 1370 (1970).

K.M. Smith et al., Nucl. Phys. B91, 45 (1975).

G.A. Akopdzhanov et al., Eur. Phys. J. C40, 343 (2005).

[2] L. Maiani and N. Paver, The second DAФNE Physics Handbook, INFN, LNF, Vol 1, 51 (1995).

E.P. Shabalin, Phys. Atom. Nucl. 68, 88 (2005).

A.A. Belkov, A.V. Lanyov and G. Bohm, Czech. J. Phys. 55 Suppl. B, 193 (2004).

G. D'Ambrosio and G. Isidori, Int. J. Mod. Phys. A13, 1 (1998).

I. Scimemi, E. Gamiz and J. Prades, hep-ph/0405204.

G. Fäldt and E.P. Shabalin, Phys. Lett. B635, 295 (2006).

[3] G. D'Ambrosio, G. Isidori and G. Martinelli, Phys. Lett. B480, 164 (2000).

E.P. Shabalin, ITEP-8-98 (1998).

[4] V. Fanti et al. (NA48), Nucl. Inst. Methods A574, 433 (2007).

[5] W.-M. Yao et al. (PDG), J. Phys. G33, 1 (2006).

[6] J.R. Batley et al., Eur. Phys. J. C52, 875 (2007). 